\title{
Preoperative Microbiological Screening in Pediatric Assessment of Skeletal Dysplasias Cases Before Planned Orthopedic Surgery
}

\author{
Milka Dikova ${ }^{1}$, Svetla Nikolova ${ }^{2 *}$, Alexandre Loukanov ${ }^{3,4}$ \\ ${ }^{1}$ University Orthopedic Hospital "Prof. Boycho Boychev", Sofia, Bulgaria; ${ }^{2}$ Department of Biology, Medical Genetics and \\ Microbiology, Faculty of Medicine, Sofia University "St. Kliment Ohridski", Sofia, Bulgaria; ${ }^{3}$ Division of Strategic Research \\ and Development, Graduate School of Science and Engineering, Saitama University, Saitama, Japan; ${ }^{4}$ Laboratory of \\ Engineering NanoBiotechnology, Department of Engineering Geoecology, University of Mining and Geology "St. Ivan \\ Rilski", Sofia, Bulgaria
}

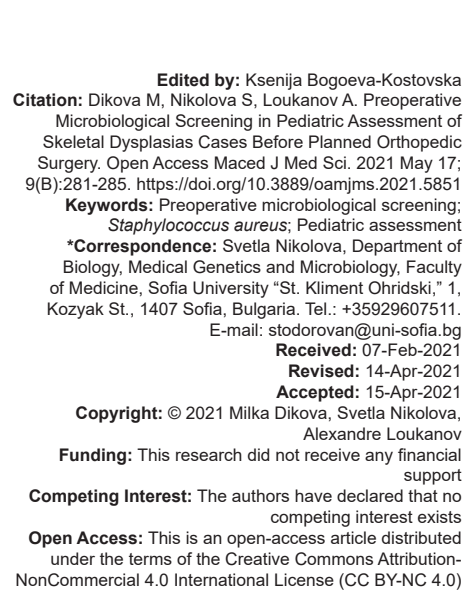

\section{Introduction}

The skeletal dysplasias are rare diseases that cause a variety of orthopedic, neurological, hearing and visual disturbances, as well as pulmonary, cardiac, and renal complications [1]. In addition, they are monogenic and manifest multiple skeletal abnormalities which are related to particular groups of bones [2]. The skeletal dysplasias were placed in 42 groups based on their clinical, radiographic, and/or molecular phenotypes in The Nosology and Classification of Genetic Skeletal Disorders from 2019 [3].

There are limited data in the medical literature on preparation for planned orthopedic surgery in these children, which require the use of general recommendations or consensus developed for adult patients. All authors recommend that the pediatric evaluation before this surgery should be based on medical history, clinical examination, and studies in a wide range of opinions. It has been established that the laboratory tests should be systematized on the basis of anamnesis and physical examination, age of the patient, and complexity of the surgical procedure. It is the physician's responsibility to evaluate the benefit/risk ratio for each specific laboratory study [4]. Goldschneider et al. (2014) suggested that clinical tests should be performed in patients with "specific" diseases to create the basis for the next stages of treatment [5]. On the other hand, Rose (2012) concluded that there was very limited data for the preoperative preparation of children with skeletal dysplasias [6]. Due to this reason, the treatment process remains a challenge which requires detailed pediatric assessment with great precision and caution, especially in the case of pre-operative risk [7]. The American Academy of Pediatrics recommend preoperative tests to be performed to determine a certain medical condition: (1) Affecting the assessment of the surgical risk; (2) reducing the surgical risk, or (3) facilitating the perioperative decision-making [8].

In the current study, we decided to include a microbiological screening approach of pathogenic microorganisms as an essential and reliable component of such pediatric assessment to reduce 
the potential perioperative risk of infections and to improve the antibacterial prophylaxis. We aim to detect staphylococcal or other transient bacterial colonization by examining nasal and throat secretions in potential carriers. For identification of the isolated bacterial strains, we mainly use the $\mathrm{BD} \mathrm{BBL}^{\mathrm{TM}}$ Crystal $^{\mathrm{TM}}$ and Thermo Scientific ${ }^{\mathrm{TM}}$ Remel $^{\mathrm{TM}}$ products and systems. The antibiotic susceptibility test was performed by the Bauer-Kirby diffusion-disk method and the minimum inhibitory concentrations (MICs) analyzed, according to Clinical and Laboratory Standards Institute (CLSI) criteria [9].

Staphylococcus aureus is a leading cause of soft tissue and bone infections in the period after orthopedic surgery. The World Health Organization recommends decolonization with mupirocin before orthopedic procedures in adult patients colonized with methicillin-resistant $S$. aureus (MRSA); no recommendations were made regarding the pediatric population [10]. Although MRSA colonized children may have lower risks of subsequent infection than adults, children who acquire MRSA in the hospital have similarly high rates of infection. Preventing transmission of MRSA in hospitalized children should remain a priority [11].

We present a preoperative microbiological protocol for pediatric assessment before planned orthopedic surgery. We apply it to rare skeletal diseases with possible involvement of other systems that change the perioperative risk.

\section{Materials and Methods}

The University's Ethics Committee approved the presented study. All patient data were stored according to the rules for protection of personal information.

\section{Patients}

The study included a total of 188 preoperative assessments of specified skeletal dysplasia cases undergoing planned orthopedic surgery within a period of 10 years (from 2004 to 2014) at the University Orthopedic Hospital. The study participants were aged between 3 months and 17 years. The pre-operative assessments of children from 3 months up to 1 year are 11 , from 1 to 3 years - 19, from 3 to 6 years - 52, from 6 to 10 years -41 , and over 10 years -65 . The gender ratio was 117 boys to 71 girls. After anamnesis and clinical examination, 29 different diagnoses from the group of genetic skeletal dysplasias were established. Anamnestic data were collected by certain rules through questionnaires with emphasis on anemia, blood transfusion, drug therapy, past, and concomitant diseases. The physical examination followed the established model. We reported height, facial and cranial dysmorphism, abnormalities in the respiratory, cardiovascular, and nervous systems the day before surgery.

The microbiological and the other laboratory tests were performed 3 and 1 day before surgery, respectively.

\section{Laboratory analysis}

We used the following laboratory parameters to complete the pediatric preoperative assessment: Hemoglobin $(\mathrm{g} / \mathrm{l})$, platelets $\left(10^{9} / \mathrm{l}\right)$, blood glucose (mmol/l), serum glutamic-oxaloacetic transaminase (U/L), serum glutamic-pyruvic transaminase (U/L), serum creatinine $(\mathrm{mmol} / \mathrm{l})$, total protein $(\mathrm{g} / \mathrm{l})$, and fibrinogen level $(\mathrm{g} / \mathrm{l})$. The tests were performed at System KH-21N Hematology Analyzer and Roche's COBAS MIRA Plus Biochemical Analyzer. The amount of hemoglobin was an essential parameter in our report, following the recommendations of SARNePI [12]. In patients with calcium/phosphorus metabolism disorders, the pre-operative screening for total calcium ( $\mathrm{mmol} / \mathrm{l})$, phosphorus (mmol/l), and alkaline phosphatase (U/I) was also conducted.

\section{Microbiological screening approach}

We analyzed nasal and throat secretions 3 days before surgery to detect carriers of $S$. aureus and/or transient carriers of pathogenic flora in these upper airway niches. We developed and applied an original scheme (algorithm) of procedures and protocols to achieve an improved isolation and determination of demanding etiological bacterial pathogens. It consists of preliminary treatment with sterile pancreatin of viscous secretions, consequent microscopic characterization, and usage of a common selective medium for Moraxella and Haemophilus, as well as a quantitative method for evaluation of the clinical significance of isolates. The kits provided by Crystal and Thermo Fisher Scientific were used to identify the isolated bacterial strains. Antimicrobial susceptibility was determined by the Bauer-Kirby diffusion-disk method and MICs analyzed, according to CLSI criteria [9].

Children with MRSA from the nasal niche were treated with $2 \%$ Mupirocin nasal ointment 5 days before surgery. Patients with MRSA colonizing their throat niche were evaluated for prophylaxis with vancomycin, $30 \mathrm{~min}$ before surgery, in a single dose. Treatment of Streptococcus pyogenes was performed and the surgical procedure was postponed until a negative result. The assessment in transient carriers was associated with antibacterial prophylaxis with firstgeneration cephalosporins or antibiotic susceptibility testing. 


\section{Statistical data processing}

Descriptive analysis and hypothesis testing were used in the presented study. The obtained results by descriptive analysis (frequency, mean, minimum, maximum, and standard deviation values) were summarized in tables. The hypothesis testing with a level of significance $<0.05$ was processed by the twotailed Fisher's Exact Test with the statistical package IBM SPSS Statistics 19.0 for Windows [13], [14].

\section{Results}

The results of pre-operative pediatric assessment in a total of 188 cases with 29 clinical diagnoses from the group of genetic diseases of the skeleton, having undergone planned orthopedic surgery for a 10-year period, are presented below.

The observed medical conditions were related to 20 of the groups from The Nosology and Classification of Genetic Skeletal Disorders from 2019.

The patients were separated into groups according to the corresponding diseases as follow: Achondroplasia $(n=3)$, spondyloepiphyseal dysplasia congenita $(n=11)$, Melnick - Needles syndrome $(n=1)$, oral-facial-digital syndrome/OFDS/(n=5), dysplasia epiphysealis multiplex $(n=15)$, dysplasia metaphysaria $(n=3)$, mesomelic dysplasia $(n=4)$, Conradi-Hünermann syndrome $(n=2)$, osteopetrosis $(n=1)$, osteogenesis imperfecta ( $\mathrm{n}=25)$, hypophosphatemic rickets $(n=6)$, hypophosphatasia $(n=3)$, alpha-mannosidosis $(n=1)$, mucopolysaccharidosis type I/MPS I/( $n=1)$, exostosis multiplex congenita $(n=19)$, McCune-Albright syndrome/MAS/(n = 6), enchondromatosis/Morbus Ollier/ $(n=6)$, multiple enchondromatosis $(n=4)$, Proteus syndrome $(n=3)$, hyaline fibromatosis syndrome/ $\mathrm{HFS} /(\mathrm{n}=1)$, cleidocranial dysplasia/CCD $/(\mathrm{n}=2)$, Apert syndrome $(n=3)$, Crouzon syndrome $(n=2)$, ischiopatellar dysplasia $(n=3)$, genitopatellar syndrome $(n=2)$, Poland syndrome ( $n=19)$, Rubinstein-Taybi syndrome $(n=1)$, and limb hypoplasia $(n=37)$, where $\mathrm{N}$ is the number of cases.

The anamnesis found anemia in 22 cases (11.7\%), pre-transfusion in 20 assessment cases (10.6\%), and drug therapy in 25 assessment cases (13.4\%). It also found an exceptionally heterogeneous group of past and accompanying illnesses in 86 cases (45.7\%). The cardiovascular system involvement including arterial hypertension, congenital heart defects, and dextrocardia was found in 15 cases (8\%). The concomitant respiratory disease group included $13.4 \%$ of the cases. Central nervous system diseases such as epilepsy and internal hydrocephalus with a ventriculoperitoneal shunt were found in $4.3 \%$ of the cases. The physical examination found dwarfism in $20.2 \%$, cranial and facial dysmorphism in $12.8 \%$, and extraskeletal manifestations from different organ systems in $36.8 \%$ of the pre-operative assessments. The results from the laboratory tests conducted within the day before the surgery are summarized in Table 1.

Table 1: Laboratory parameters obtained by the clinical tests, № - number of pre-operative assessment cases

\begin{tabular}{llllll}
\hline Laboratory parameters & № & Min & Max & Mean & SD \\
\hline Hemoglobin $(\mathrm{g} / \mathrm{l})$ & 164 & 62.00 & 154.00 & 127.50 & 13.15 \\
Platelets $\left(10^{9} / \mathrm{l}\right)$ & 161 & 33.00 & 561.00 & 314.45 & 85.63 \\
Creatinine $(\mu \mathrm{mol} / \mathrm{l})$ & 148 & 17.00 & 81.0 & 51.85 & 13.05 \\
Blood glucose $(\mathrm{mmol} / \mathrm{l})$ & 150 & 3.54 & 6.40 & 4.90 & 0.59 \\
SGOT $(\mathrm{U} / \mathrm{L})$ & 154 & 6.00 & 76.00 & 22.14 & 11.61 \\
SGPT $(\mathrm{U} / \mathrm{L})$ & 153 & 11.00 & 109.00 & 29.23 & 11.77 \\
Protein $(\mathrm{g} / \mathrm{l})$ & 128 & 54.00 & 86.00 & 70.60 & 6.41 \\
Fibrinogen $(\mathrm{g} / \mathrm{l})$ & 126 & 1.30 & 5.50 & 3.12 & 0.85 \\
Calcium $(\mathrm{mmol} / \mathrm{l})$ & 17 & 1.75 & 2.82 & 2.36 & 0.28 \\
Phosphorus $(\mathrm{mmol} / \mathrm{l})$ & 17 & 0.60 & 1.95 & 1.23 & 0.42 \\
Alkaline phosphatase $(\mathrm{U} / \mathrm{L})$ & 15 & 92.00 & 910.00 & 426.66 & 213.86 \\
\hline SGOT: Serum glutamic-oxaloacetic transaminase, SGPT: Serum glutamic-pyruvic transaminase.
\end{tabular}

We analyzed the results from the microbiological studies on a total of 246 secretions - 122 nasal and 124 throat samples. In $50.8 \%$ of the cases, we observed normal microflora, and $49.2 \%$ of all cases carried potentially pathogenic microflora as summarized in Table 2.

Table 2: Microbiological analysis of the upper respiratory tract niches, № - number of preoperative assessment cases

\begin{tabular}{llll}
\hline Niche & Microflora & № & $\%$ \\
\hline General & Normal microflora & 63 & 50.8 \\
& Carrier & 61 & 49.2 \\
Nasal niche & Total & 124 & 100.0 \\
& Normal microflora & 68 & 55.7 \\
& MSSA & 48 & 39.3 \\
& MRSA & 4 & 3.3 \\
& Streptococcus pneumoniae & 2 & 1.6 \\
Throat niche & Total & 122 & 100.0 \\
& Normal microflora & 96 & 77.4 \\
& MSSA & 21 & 16.9 \\
& MRSA & 2 & 1.6 \\
& Streptococcus pyogenes & 1 & 0.8 \\
& Candida albicans & 1 & 0.8 \\
& Moraxella catarrhalis & 3 & 2.4 \\
& Total & 124 & 100.0 \\
\hline MSSA: Methicillin-susceptible S. aureus; MRSA: Methicillin-resistant S. aureus.
\end{tabular}

Figure 1 shows methicillin-susceptible $S$. aureus (MSSA) detected in 69 and MRSA in 6 evaluations.

\section{Discussion}

Following the recommendation of the Italian Society of Pediatric and Neonatal Anesthesia and Intensive Care from 2014, the amount of hemoglobin

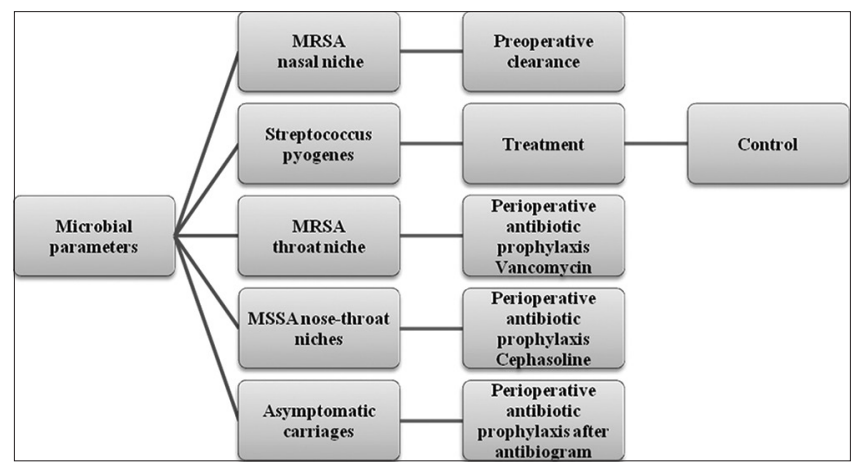

Figure 1: Pre-operative microbiological analysis and perioperative antibacterial prophylaxis 
was an essential parameter in our report, because of the type of surgery and expected blood loss, as well as a preoperative basis of the applied transfusion strategy [12]. The obtained clinical laboratory data were normal in $81 \%$ of the preoperative assessment, and abnormal in $19 \%$, where deviations in the hemoglobin values were observed in $13.8 \%$ of the cases (Table 1 ).

There was a statistically significant difference in colonization with pathogenic microflora such as MSSA, MRSA, Streptococcus pneumoniae, S. pyogenes, Moraxella catarrhalis, and Candida albicans when comparing the upper airway niches $(44.3 \%$ for nasal niche vs. $22.6 \%$ for throat niche, $p=0.0004$, Fisher's Exact Test) as well as in colonization with $S$. aureus strains only $(42.6 \%$ for nasal niche vs. $18.5 \%$ for throat niche, $p<0.0001$, Fisher's Exact Test). Colonization is greater in the nasal niche and the predominant pathogenic species in both niches is $S$. aureus (Table 2).

In the case of nasal niche, infections with S. aureus were identified in $39.3 \%$ of the results as methicillin sensitive strains (MSSA) and in 3.3\% as methicillin-resistant strains (MRSA). In a study of 247 children undergoing varus derotation osteotomy, Nazareth et al. (2020) found 2\% positive MRSA swab tests. The MRSA nasal swab test is routinely used as a pre-operative screening method based on the assumption that the results will influence the treatment management and decrease the surgical site infections rates [15]. To determine pre-operative MRSA colonization status, Nielsen et al. (2018) presented a retrospective chart review of patients after posterior spinal fusion performed between 2004 and 2014, and identified 2.3\% MRSA-positive cases [16].

Patients whose upper airways are colonized are 2-9 times more at risk for staph infections after surgical procedures than those who are not. This result was supported also by other reports [17]. Administration of Mupirocin reduces the risk of postoperative $S$. aureus infections up to 5 times in patients who have been colonized before surgery. This was demonstrated in a study of 614 adult patients undergoing major orthopedic surgery [18].

We developed a preoperative microbiological protocol to specify the staphylococcal carrier in the presented patients for orthopedic planned surgery.

Patients with MSSA nasal and throat colonization undergo antibacterial prophylaxis with first generation cephalosporins (Figure 1). Pre-operative eradication of MRSA carriage in four cases of nasal colonization and vancomycin prophylaxis in two cases of throat colonization were performed. We treated S. pyogenes in one patient and did not subject the patient to elective surgery. In two cases of transient nasal carriage of pathogenic microflora and in three cases of transient throat carriage, we performed perioperative prophylaxis in accordance with the determined antibiotic sensitivity.
Hadley et al. (2010) performed screening of 1644 patients undergoing primary total knee or total hip arthroplasty using a working protocol for adults and found $3.5 \%$ positive nasal swabs for MRSA [19].

\section{Conclusion}

The reported study shows a pre-operative analytical approach when performing a pediatric assessment for planned orthopedic surgery with the use of a microbiological work protocol. It is performed in children with regular skeletal diseases that have concomitant deviations detected by the identification systems. Laboratory parameters in a high percentage of cases are normal and the deviations are associated with the main diseases or causes related to them. The microbiological work protocol detects staphylococcal carriers, provides a solution for eradication of MRSA and the possibility of antibacterial prophylaxis consistent with antibacterial susceptibility.

\section{References}

1. KrakowD. Skeletal dysplasias. Clin Perinatol. 2015;42(2):301-19. PMid:26042906

2. Hall BK, Miyake T. All for one and one for all: Condensations and the initiation of skeletal development. Bioessays. 2000;22(2):138-47. https://doi.org/10.1002/ (sici)1521-1878(200002)22:2<138::aid-bies5>3.0.co;2-4 PMid: 10655033

3. Mortier GR, Cohn DH, Cormier-Daire V, Hall C, Krakow D, Mundlos S, et al. Nosology and classification of genetic skeletal disorders: 2019 revision. Am J Med GenetA. 2019;179(12):2393419. https://doi.org/10.1002/ajmg.a.61366 PMid:31633310

4. Roizen MF, Foss JF, Fisher SP. In: Miller RD, editor. Preoperative Evaluation. $5^{\text {th }}$ ed. New York: Churchill Livingstone; 2000. p. 824-83.

5. Goldschneider KR, Cravero JP, Anderson C, Bannister C, Hardy C, Honkanen A, et al. The pediatrician's role in the evaluation and preparation of pediatric patients undergoing anesthesia. Pediatrics. 2014;134(3):634-41. PMid:25157004

6. Rose A. Pediatric Otolaryngology Challenges in Multi-system Disease, an Issue of Otolaryngologic Clinics. Vol. 45-3. Philadelphia, PA: Elsevier Health Sciences; 2012. p. 110-34.

7. White KK, Bompadre V, Goldberg MJ, Bober MB, Cho TJ, Hoover-Fong JE, et al. Best practices in peri-operative management of patients with skeletal dysplasias. Am J Med Genet A. 2017;173(10):2584-95. https://doi.org/10.1002/ ajmg.a. 38357

PMid:28763154

8. Mclnerny TK, Adam HM, Campbell, DE. Textbook of Pediatric Care. $2^{\text {nd }}$ ed. Itasca: American Academy of Pediatrics; 2016.

9. Gergova R, Minchev P, Dikova M, Gergov S, Jurova C, llieva T, 
et al. Bacterial spectrum of etiology of the community-acquired respiratory infections in childhood and antimicrobial resistance. Bulg Med J. 2013;7(2):45-54.

10. Allegranzi B, Bischoff $\mathrm{P}$, De Jonge S, Kubilay NZ, Zayed B, Gomes SM, et al. New WHO recommendations on preoperative measures for surgical site infection prevention: An evidencebased global perspective. Lancet Infect Dis. 2016;16(12):27687. https://doi.org/10.1016/s1473-3099(16)30398-x PMid:27816413

11. Milstone AM, Goldner BW, Ross T, Shepard JW, Carroll KC, Perl TM. Methicillin-resistant Staphylococcus aureus colonization and risk of subsequent infection in critically ill children: Importance of preventing nosocomial methicillin-resistant Staphylococcus aureus transmission. Clin Infect Dis. 2011;53(9):853-9. https:// doi.org/10.1093/cid/cir547

PMid:21878424

12. Serafini $G$, Ingelmo PM, Astuto M, Baroncini S, Borrometi $F$, Bortone L, et al. Preoperative evaluation in infants and children: Recommendations of the Italian Society of Pediatric and Neonatal Anesthesia and Intensive Care (SARNePI). Minerva Anestesiol. 2014;80(4):461-9.

PMid:24193177

13. Nikolova S, Dikova M, Dikov D, Djerov A, Savov A, Kremensky I, et al. Positive association between a polymorphic locus near the LBX1 gene and predisposition of idiopathic scoliosis in Southeastern European population. J Appl Biomed. 2019;17:184-9. https://doi.org/10.32725/jab.2019.011

14. Nikolova S, Dikova M, Dikov D, Djerov A, Savov A, Kremensky I, et al. Positive association between the progression of idiopathic scoliosis and the common variant near the LBX1 gene in Southeast European population. J Appl Biomed.
2018;16(4):344-9. https://doi.org/10.1016/j.jab.2018.07.001

15. Nazareth A, Bains S, Andras L, Goldstein R, Kay R. Pre-operative methicillin resistant Staphylococcus aureus results do not predict surgical site infections in children undergoing varus derotational osteotomy. Medicine (Baltimore). 2020;99(26):e20517. https:// doi.org/10.1097/md.0000000000020517

PMid:32590733

16. Nielsen E, Andras L, Harris L, Skaggs D. Methicillin-resistant Staphylococcus aureus swab results did not change treatment or outcome in pediatric spinal fusion patients. J Am Acad Orthop Surg. 2018;26(17):617-23. https://doi.org/10.5435/ jaaos-d-17-00010

PMid:30036217

17. Yano K, Minoda Y, Sakawa A, Kuwano Y, Kondo K, Fukushima W, et al. Positive nasal culture of methicillin-resistant Staphylococcus aureus (MRSA) is a risk factor for surgical site infection in orthopedics. Acta Orthop. 2009;80(4):486-90. https://doi.org/10.3109/17453670903110675 PMid:19593719

18. Kalmeijer MD, Coertjens $H$, van Nieuwland-Bollen $P M$, Bogaers-Hofman D, de Baere GA, Stuurman A, et al. Surgical site infections in orthopedic surgery: The effect of mupirocin nasal ointment in a double-blind, randomized, placebocontrolled study. Clin Infect Dis. 2002;35(4):353-8. https://doi. org/10.1086/341025

PMid:12145715

19. Hadley S, Immerman I, Hutzler L, Slover J, Bosco J. Staphylococcus aureus decolonization protocol decreases surgical site infections for total joint replacement. Arthritis. 2010;2010:924518. https://doi.org/10.1155/2010/924518 PMid:22046511 